\title{
Asleep at the Wheel: A Case for a Gastrointestinal (GI) Adenocarcinoma Early Detection Algorithm
}

\author{
Rhonda Robert* \\ Department of Pediatrics, Anderson Cancer Center, Houston, USA
}

*Corresponding author: Rhonda Robert, Department of Pediatrics, Anderson Cancer Center,Unit 0087,RoomB8.4409,1515

Holcombe Boulevard, Houston, TX 77030-4009, USA

\begin{tabular}{|c|c|}
\hline ARTICLE INFO & ABSTRACT \\
\hline Received: 慧 September 05, 2020 & Citation: Rhonda Robert. Asleep at the Wheel: A Case for a Gastrointestinal (GI) \\
\hline Published: 幽 September 16, 2020 & $\begin{array}{l}\text { Adenocarcinoma Early Detection Algorithm. Biomed J Sci \& Tech Res 30(3)-2020. BJSTR. } \\
\text { MS.ID.004961. }\end{array}$ \\
\hline
\end{tabular}

\section{Introduction}

As a young professional, I practiced psychology in a pediatric burn intensive care unit. When the traumatized children displayed symptoms of anxiety, the typical staff response suggested the staff felt helpless. Once an anxiety management protocol was developed and implemented, the staff acted swiftly and completely to ameliorate the child's suffering. I flashed back to this experience twenty plus years later, when I was practicing psychology in a major cancer center and my husband was diagnosed with cholangiocarcinoma (CCA). This initial clinical diagnosis was followed with a biopsy-based diagnosis of moderately to poorly differentiated adenocarcinoma, and 2 months thereafter a clinical diagnosis of gallbladder cancer. Furtively exploring what this meant, I learned from the American Cancer Society (ACS) that these disease are fatal and possible disease risk factors included obesity, smoking, and poor dietary practices (deficiencies in vegetables, legumes, and grains), and sedentary lifestyle. However, my husband was of normal weight, exercised 3 to 5 hours weekly, had an active lifestyle, and ate an Atkins style diet. Most meals consisted of a protein and vegetables. Groceries were whole, organic foods. He subscribed to food choice principals purported in movies such as Food Inc. The household was free of corn syrup. The fowl was all natural; beef was grass fed; the fish was local and wild-caught; and country of food's origin was highly selective. He was a longtime community supported agriculture (CSA) group member and my husband was on the group's organizing committee.

Seeing that "anything but" was true in our situation, this suggested that the scientific knowledge base about these diseases was nil, and useless information dragged up from the dark ages was used to assuage our helplessness. Ultimately, we need the systemic answers and rally behind the geniuses who develop targeted drugs, immune therapies, surgical wonders, and radiation disease eradicators! Immediately, however, we could improve the timeliness of disease detection. At present, fewer than 1 in 10 persons are diagnosed early enough to have curative surgical resection. My husband, may his memory be a blessing, was examined by a full professor of internal medicine who was boarded in family medicine and practicing at a major academic medicine affiliated institution in the world's largest medical center, days before his death. The stage IVb, T2b, N1, M1 wildly metastatic cancer which involved the gallbladder, bile ducts, retroperitoneal lymph nodes, lungs, and omentum went undetected. What might have tipped the examiner, and numerous other medical providers months and years prior to find this alien of death? Systematic utilization of medical and family history, diagnostic tests (blood, ultrasound, scan) and prophylactic cholecystectomy.

The related literature, what little there was, did not mirror that which was reported by CCA Foundation's Support Group members who are comprised of patients, family members, and their advocates. Why might this be? The health decline and death are so rapid that the diseases' victims may never get to a physician, let alone a proper diagnosis. Let us just take the estimated prevalence rates. These diseases are supposedly rare, meaning a couple thousand in the United States annually. However, within my personal friendship network (omitting medical professional colleagues and other 
patients encountered in the course of my husband's treatment), 5 have died of CCA in the last year (HL's sister; officiant's mentor; DM's friend; CSA member's father-in-law; CE employee's daughter's father-in-law). If I were to liberally estimate the total number in my friendship network, the count may be as high as 300 , meaning that the annual prevalence rate in this sample would approximate $1.7 \%$. Also unlikely is that 5 of the estimated 2,000 to 3,000 persons diagnosed with CCA annually in the United States are in my person to person social network. Given that these 5 people had substantive graduate school education, moderately to high SES, lived most their lives within blocks of major world class medical centers which included a comprehensive cancer center, and were fully engaged in health-promoting behavior and prophylactic, adherent medical service seekers, the current best estimate of CCA prevalence is highly suspected to be a gross underestimate.

I would like to use my husband's medical, family, and diagnostic history to illustrate the need for:

a) A validated patient-reported screening measure,

b) A best practice diagnostic protocol for ruling out CCA and gallbladder cancer; and

\section{c) Prophylactic cholecystectomy.}

I will report the history in terms of a timeline, beginning with the oldest information available, referred to in amount of time that predated my husband's CCA diagnosis, at age 55 years of age:

a. Minus 40 years - 39-year-old mother, diagnosed with gallstones and high cholesterol, had cholecystectomy.

b. Minus 24 years - 28-year-old sister diagnosed with carcinoid syndrome, abdominal neuro-endocrine tumors.

c. Minus 23 years - 42-year-old maternal aunt died of colon cancer.

d. Minus 19 years - Maternal grandmother died of gallbladder cancer within 3 months of diagnosis.

e. Minus 15 years - 37-year-old sister diagnosed with gallstones and had cholecystectomy.

f. Minus 11 to 14 years - Patient diagnosed with gallstones and advised to avoid cholecystectomy because

1. Surgeries have risks, which in this case was $0.4 \%$ risk of death.

2. The gallbladder serves a useful, though unessential function; and

\section{Gallbladder malignancies are rare.}

Emergency medical services for retroperitoneal pain, treated for kidney stones. Outpatient medical servicefor abdominal pain, treated for peptic ulcer.Severe, frequent constipation and diarrhea. a. Minus 5 years - Patient diagnosed with diabetes, asymptomatic, required insulin,

b. Minus 3 years - Outpatient medical services for retroperitoneal/flank pain and blood in urine.

c. Minus 2 years - Specialized outpatient medical services (urologist) for blood in the urine and intermittent abdominal pain.

d. Minus 3 to 6 months - Subtle health changes: loss of muscle mass, change in hair quality/texture; retroperitoneal and abdominal pain; irritability and fatigue; atypical behaviors / cognitive errors, one of which resulted in a major automobile collision (with a lifetime history of 0 vehicular accidents); premature satiety.

e. Minus 10 days - Outpatient medical services for abdominal pain.

\section{Patient Reported Screening Measure}

If just this one persons' complaint were to be compiled, patient reported screening items would be numerous. Personal history would include gallstones, retroperitoneal/flank/abdominal pain, blood in urine, constipation, diarrhea, diabetes, loss of muscle mass, hair thinning or change in hair quality/texture, irritability, fatigue, premature satiety, and subtle behavioral/cognitive changes. Family history would includegallstones,gallbladder cancer, cholangiocarcinoma,carcinoid syndrome, colon cancer, cholecystectomy. As a matter of fact, 4 of these very items have been established as increasing one's risk for GI adenocarcinoma $[1,2]$. Benign gallbladder disease (including gallstones) increases risk as much as 34.4 times; family history of benign gallbladder disease up to 3.6; family history of GC, 1st degree relative up to 13.9; and personal history of constipation. Are we asleep at the wheel when we hear GI complaints? Do we write the person off as "psychosomatic"? Do we assume a "la crise de foie" (crisis of the liver) and consequently minimize the complaint? We will not know unless we initiate systematic screening for commonly reported GI complaints and thoroughly query personal and family history with a structured, forced choice written assessment in which the items include scientifically substantiated correlates of GI malignancies.

\section{Best Practice Diagnostic Protocol}

For 20 years, my husband's physicians primarily relied on presenting concern, physical exam, and an occasional blood test. Tools for disease surveillance could have been employed and were not:

a) For a blood test result to be helpful, the correct tests must be run and the results must be addressed. LFT's, Carcinoembryonic antigen (CEA), and CA19-9 may be very helpful in detecting these cancers. Of note, positive test results are commonly assumed and not further investigated. 
b) Ultrasonography

c) Endoscopic ultrasonography

d) CT, MRI and MR cholangiopancreatography (MRCP)

\section{Prophylactic cholecystectomy}

Prophylactic cholecystectomy should be in the treatment algorithm.An on-line cancer research organization in the UK reports a 60-fold increase in gallbladder cancer for persons with both gallstones andfamily history of gallstones. And a 5-fold risk increase amongst 1st degree relatives with gallbladder cancer.

Epidemiologically, a correlational relationship has been established between number of cholecystectomies and rates of gallbladder cancer. In large populations in which cholecystectomy was increasingly prevalent, the occurrence of gallbladder cancer incrementally decreased [3,4].For example, in the 1980, Chile's increase of GC mortality correlated with the decrease in number of cholecystectomies [1].Another correlation has been established between the number of years a person lives with gallstones and that person developing gallbladder cancer. The greater the number of years a person lives with gallstones, the higher one's risk for gallbladder cancer.

\section{In Summary}

In 1983, while on an Egyptian archeological tour and learning about Tutankhamun in the Valley of the Kings, I remember the tour guide impressing upon the group members the mysterious, unexplainable premature death of this young teenage pharaoh. Fast forward to 2012 and the Museum of Fine Arts Houston exhibit in which King Tut was featured. Modern medical technology, including a CT scan, shed new light on Tut's health and possible cause of death. I was amazed that so much could change in my own lifetime, more specifically between the span of my early and middle adult years. The gastro-intestinal adenocarcinoma victims are too ill to advocate for themselves. By the time they know what has struck, their lives are exhausted. I believe lives can be saved through the practice of systematic risk factor and symptom assessment, utilization of disease detection/diagnostic tools, and prophylactic cholecystectomy. Please join the campaign and advocate for these changes.

\section{References}

1. G Randi, Silvia F, Carlo LV (2006) Gallbladder cancer worldwide: Geographical distribution and risk factors. Int. J. Cancer 118(7): 15911602.

2. Yagyu K, Lin Y, Obata Y, Kikuchi S, Ishibashi T, et al. (2004) Bowel movement frequency, medical history and the risk of gallbladder cancer death: a cohort study in Japan. Cancer Sci 95(8): 674-678.

3. Wood R, Fraser LA, Brewster DH, Garden OJ (2003) Epidemiology of gallbladder cancer and trends in cholecystectomy rates in Scotland, 1968-1998. Eur J Cancer 39(14): 2080-2086.

4. Diehl AK, Beral V (1981) Cholecystectomy and changing mortality from gallbladder cancer. Lancet 318(8239): 187-189.

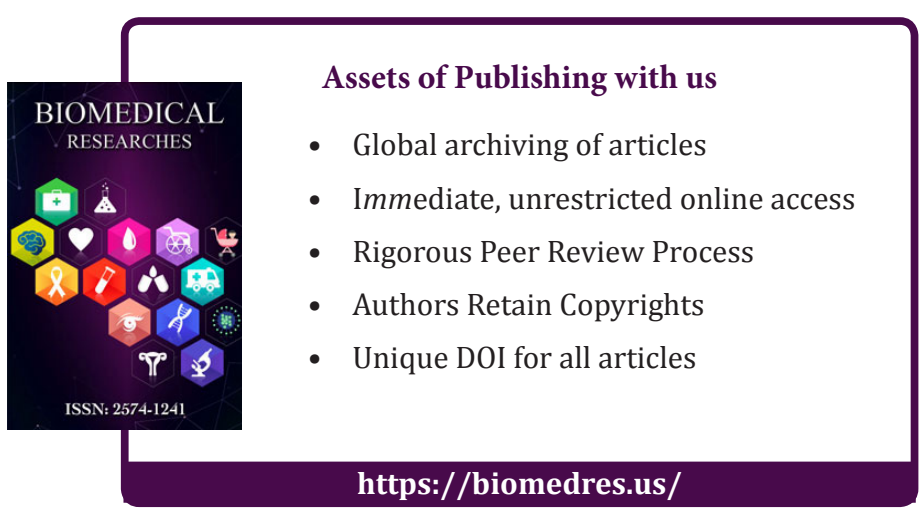

